\title{
Practice makes perfect: An adaptive active learning framework for image classification
}

\author{
Zhipeng Ye, Peng Liu, Jiafeng Liu, Xianglong Tang, Wei Zhao \\ Harbin Institute of Technology, No. 92 West Dazhi Street, Harbin 150001, China
}

\begin{abstract}
Active learning is an effective method for iteratively selecting a subset of images from an unlabeled dataset. One of the most widely used active learning strategies is uncertainty sampling. However, traditional sampling strategies do not take the category of samples into consideration, and the selected images do not reflect the desired training distribution, leading to the result that additional labeling work needs to be done. To deal with these problems, from the aspect of visual perception, we improve the traditional entropy-based uncertainty sampling strategy by introducing a certainty measurement estimated by a bag-of-visual-words (BoVW). The Rescorla-Wagner perceptive model is utilized to quantify the stop criterion. This method differs from previous approaches that treated sampling and classifying process separately: we treat the learning process as a uniform model by proposing a new evolving sample selection method that uses the unified negative-accelerated learning principle and takes category distribution into consideration. A classifier is trained to provide category distributions for the sampling process to improve its sampling performance and reduce additional annotation costs for the human annotator. During the training process, weights for both modules are adaptively initialized by the inner similarity of sample set measured by structural similarity (SSIM), and dynamically adjusted according to the learning process of the human. In addition to the regular tests that are commonly utilized by traditional sampling methods, the transfer test, based on transfer learning theory, is utilized to further evaluate the performance of different sampling strategies. Experimental results on real world datasets show that our active sampling framework outperforms both baseline and state-of-the-art adaptive active learning strategies.
\end{abstract}

\section{(C) 2011 Published by Elsevier Ltd.}

Keywords: Active learning, entropy-based sampling, image classification, bag-of-visual-words, cognitive model

\section{Introduction}

Image classification is an important problem in computer vision and machine learning. Because of the explosive growth of imaging techniques, especially the fast popularization of smart devices, automatically obtaining semantic image information has become indispensable and has a wide range of applications in real-world information systems. A noteworthy problem in practice is that it is relatively easy to get unlabeled data, but labeling is expensive. Traditional supervised learning methods do not capture this distinction, motivating the field of active learning, which aims to train an accurate prediction model with minimum cost by labeling the most informative instances.

A typical active learning framework consists of two parts: a learning engine and a sample selection engine. In each iteration, the learning engine trains a model to predict the labels of unlabeled samples based on the training set. The sample selection engine selects the most effective unlabeled samples based on a certain strategy. For each annotation iteration, a subset of instances is carefully chosen for annotation by an annotator and the remaining instances remain unlabeled. The goal of active learning is to train a classifier with the labeling instances selected by active sampling 
methods. As summarized in Fig. 1, for active learning, there are three main categories: (i) membership query synthesis [1], (ii) stream-based selective sampling [2], and (iii) pool-based sampling [3]. Membership query synthesis is reasonable for many problems; however, this comes at the cost of discarding the distribution of samples. It is also time-consuming and awkward for a human annotator. The stream-based selective sampling strategy scans each unlabeled instance from source data one at a time for the learner to query. Although it overcomes the shortcoming of the membership query synthesis strategy, a threshold for the quantity of information still needs to be tuned, which is not adaptive for different scenarios. The pool-based sampling method assumes that both a small set of labeled data and a large pool of unlabeled data are available, and it has been successfully used in image retrieval and classification $[4,5]$.

For the most widely used pool-based sampling method, there are three main developed query strategies [6]: (i) uncertainty sampling, (ii) query-by-committee, and (iii) optimization models. Uncertainty sampling is fit for most classifiers with good precision and generalization ability [7] according to different forms of uncertainty measurement functions. Of these, information entropy [8] is the most commonly used. Query-by-committee is a well-known strategy that seeks a few ambiguous instances to find the best model with a small set of hypotheses. Boosting and bagging methods are commonly used to build up a committee [9]. The Kullback-Leibler divergence [10] is mostly used to evaluate the divergence of each member in the committee [11]. An optimization model is a general query strategy containing a set of query optimization functions, including "expected gradient length" (EGL) [12], loss minimization [13], and variance reduction [14].

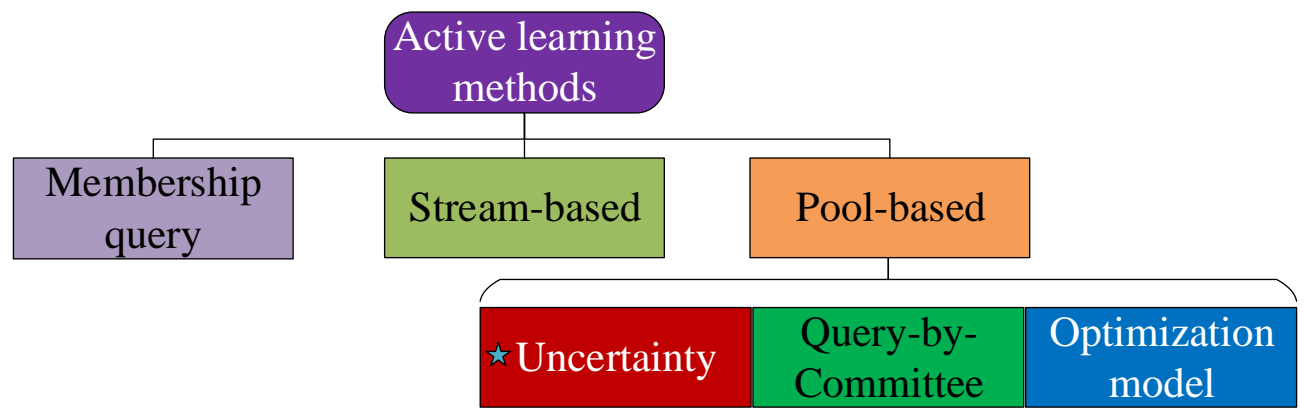

Figure 1. Main active learning scenarios. Uncertainty sampling is the approach used in this study.

Traditional entropy-based methods only consider the uncertainty of samples and ignore their category distribution, which restricts the quality of sampling. Thus, in this paper, we focus on obtaining a more accurate classifier by improving the quality of sampling and reducing the re-annotation work for the human annotator. The negativeaccelerated adaptive active sampling with a bag-of-visual-words (NAASB) framework is proposed by introducing a certain measurement into the traditional strategy to deal with this problem. The proposed sampling framework contains two parallel and interactive subtasks, image classifier training and sample selection:

(a) Image classifier training: A labeled set is utilized to train an image classifier and adaptively initialize the parameters of NAASB according to the features of different categories. Then, for each iteration, samples are selected using an active learning strategy and annotated to train and improve the performance of the image classifier. The stop criterion for the iteration is measured by the performance of the classifier according to the negative-accelerated principle.

(b) Sample selection: In every round of active learning, the certainty output of the classifier is introduced to take category distribution into consideration for entropy-based active sampling strategy. Meanwhile, the selected samples are annotated by the image classifier to reduce the work of the human annotator.

The rest of the paper is organized as follows. Section 2 summarizes the related work. In Section 3, we describe and explain the proposed NAASB framework in detail. Experimental results are given in Section 4. Finally, we offer the conclusions in Section 5. 


\section{Related Work}

In this section, we review related methods, including uncertainty sampling strategy in active learning, the bag-ofvisual-words $(\mathrm{BoVW})$ method in image classification, and the negative-accelerated learning principle provided by the Rescorla-Wagner model.

\subsection{Uncertainty sampling strategy}

Uncertainty sampling [15] is one of the most common frameworks that employ probabilistic models to evaluate the potential of instances. The prediction result of a single instance is represented by a vector, whose elements are the posterior probability with respect to each class label. Generally, the informativeness of a sample can be assessed by using the uncertainty of the instances based on models trained from the current labeled sample set. If the uncertainty of a sample is high, it implies that the current model does not have sufficient knowledge to classify the sample, and it is helpful for this sample to be contained in the training set to improve the underlying model. The uncertainty metric is designed based on individual instance importance for an accurate model construction. Applying a traditional uncertainty criterion means that the most uncertain samples should be selected. This strategy is straightforward for both probabilistic and non-probabilistic learning models, and can be divided into three main substrategies [16]: least confidence [17], sample margin [18], and entropy [8]. When using a probabilistic model for binary classification, uncertainty sampling simply queries the instances whose posterior probability of being positive is nearest to 0.5 [3], while for non-probabilistic classifiers, classifiers such as decision trees [15] and nearest-neighbors [19] are utilized by allowing each neighbor to vote for the class label of the selected instance. The uncertainty criterion has been widely explored because of its simplicity. The complexity of the uncertainty sampling strategy is $O(n)$, where $n$ is the scale of the unlabeled pool. The deficiency of the least confidence and sample margin strategies is that they ignore the output label distributions for the remaining classes, while entropy is a typical metric that represents the uncertainty over the whole output prediction distribution.

\subsection{Image classification techniques}

Image classification has been an active research field over the past several decades. There have been extensive research efforts to develop effective visual object recognizers, such as object and scene classification [20, 21], video event detection [22], and action recognition [23]. Nowadays, bag-of-features (BoF) [24] based classification is one of the most classic and commonly used approaches in image retrieval and scenario classification, whose simplicity and effectiveness have been tested over the years. Inspired by BoF, BoVW [25] was proposed to classify images. Visual words are first obtained by k-means clustering local features, commonly described by difference of Gaussians (DoGs) or Scale-invariant feature transforms (SIFTs), and the clustering centers are considered to be the base of the BoVW to represent the whole image for training and testing. However, there are several drawbacks to BoVW: i) spatial relationships are ignored between image patches during the construction of the visual vocabulary [26], ii) the hardassignment strategy of k-means does not necessarily generate an optimized visual vocabulary [27], and iii) semantics are ignored during the clustering process [28].

Many studies have been carried out to solve these problems. Chai [29] utilized foreground segmentation to improve classification performance on weakly annotated datasets. To address the fact that spatial information among local features was ignored, spatial pyramid matching (SPM) [30] was proposed to make use of spatial information for object and scene categorization. In addition, there are several ways to generate a rich descriptive visual vocabulary. Wang presented a simple but effective coding scheme called locality-constrained linear coding (LLC) in place of the vector quantization (VQ) coding in traditional SPM to improve the categorization performance [31]. Germert introduced ambiguity into visual words to improve their descriptive ability [32]. Semantic layers were constructed to narrow semantic gaps between lower features and semantic concepts to generate a more optimized visual vocabulary [33]. Lu developed a framework to identify high-level concepts with small semantic gaps from a large scale web image dataset [34]. Deng proposed a similarity-based learning approach to exploit hierarchical relationships between semantic labels at the training stage [35]. Wu proposed a semantics-preserving bag-of-words (SPBoW) scheme to learn optimized bag-of-words models by generating a semantics preserving codebook [36].

Traditional image retrieval approaches operate under the major assumption that the training and testing data are from the same domain. However, this assumption is not always valid in practice. It has been reported that the performance of traditional image classification methods is sensitive to different datasets [37] because a classifier 
may receive various kinds of input data, and the availability of human labeled training data is limited, leading to the result that it is hard for traditional approaches to model those unexpected data. Thus, there is some research targeting the knowledge transfer problem between different datasets, which is called transfer learning [38]. Transfer learning address the problems of applying knowledge of related data in different domains, which can be considered as a special learning paradigm, where partial/all training data used is under a different distribution than the testing data [39]. According to various situations, transfer learning can be roughly divided into several approaches: 1) zeroshot and one-shot learning [40, 41], 2) knowledge and visual attribute transfer [42], and 3) domain adaptation [43]. Zero and one shot learning seek to learn knowledge about a category without any instances or only a very few of them. Attribute-based representation is an interpretable level of indirections between object classes, which can be shared and reused among object classes. For knowledge and visual attribute transfer, well-designed visual attributes of unseen classes are utilized to classify unseen instances. Domain adaption is designed for a situation when there is a lot of training data in one domain but little or none in another. There are two distinctions needed in domain adaption: labeling adaption and instance adaption [44]. Labeling adaption models the changes of labeling function while instance adaption describes the variation on instance probability. Using the principle of transfer learning, in this paper, the experimental datasets are organized and divided into two aspects: regular and transfer testing to fully evaluate the performance of different sampling strategies.

\subsection{Negative-accelerated learning principle}

To achieve the goal of making the way a machine works more human-like, the introduction of a perceptual model into traditional computer vision algorithms is a great help and is able to further improve the performance of classification $[45,46,47]$. For traditional active learning strategies, iteration stops when the performance meets the predefined conditions, commonly a pre-defined threshold for the number of iterations [48, 49]. There are a few universal frameworks that simultaneously describe the parameter selection and performance changes during the learning process. Here, we introduce the famous negative-accelerated learning principle provided by the Rescorla-Wagner model [50]. In psychology, it shows extremely good performance on psychological phenomenon, including reasoning causality, social psychology, and the learning psychology of human being for classification [51]. The model was originally described as

$$
\Delta V_{n}=\alpha \gamma\left(\lambda-V_{A X}\right)
$$

where $\Delta V_{n}$ is the performance change of a classifier after the $n$-th iteration, $\alpha$ and $\gamma$ are the parameters of conditioned stimulus and unconditioned stimulus (US), respectively, $\lambda$ is the maximum conditioning that US produces and represents the limit of learning. In addition, $V_{A X}$ is the total associative strength under previous compound stimuli. The more annotation and training we do, the lower $\Delta V_{i}$ will be, i.e., the increment in performance will decrease as iterations proceed. A toy example is shown in Fig. 2. From the figure, we can see that the negative-accelerated principle describes the states of knowledge from undersaturation to saturation.

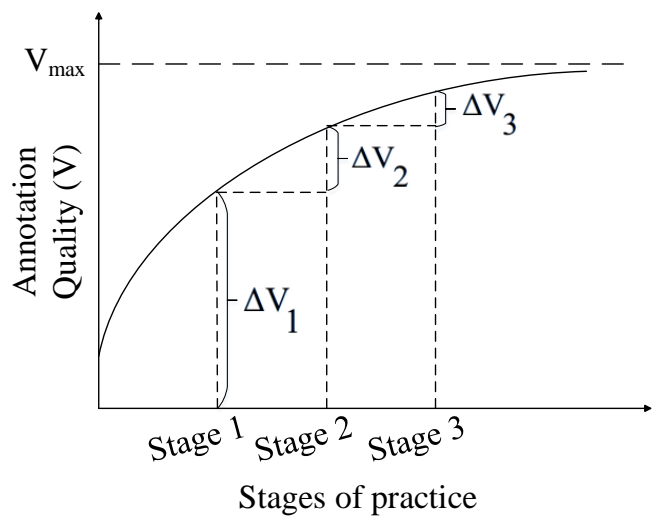

Figure 2. Curve representing the process of negative-accelerated learning. Parameter $V_{\text {max }}$ is the maximum performance a classifier may achieve and can be simply set to $100 \%$, or some other value according to practice. The overall learning trend is robust to the change of $V_{\text {max }}$. 


\section{Proposed Adaptive Active Sampling Framework}

Active learning is close to supervised learning, except that training data are not independent and identically distributed variables. Some of them are added to the training set by a dedicated process. However, traditional active sampling strategies do not take category distribution into consideration at the sampling stage and therefore restrict the sampling performance [52].

To clearly show the motivation of our work, the difference between traditional and the proposed NAASB sampling strategies is shown in Fig. 3. We can see that traditional entropy-based active learning method in sample selection ignores the category distribution, restricting the performance of the classifier during the learning iteration, while our method can generate a more precise classifier by introducing the category distribution information.

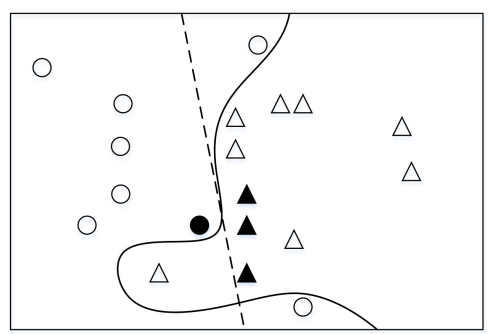

(a) Original dataset.

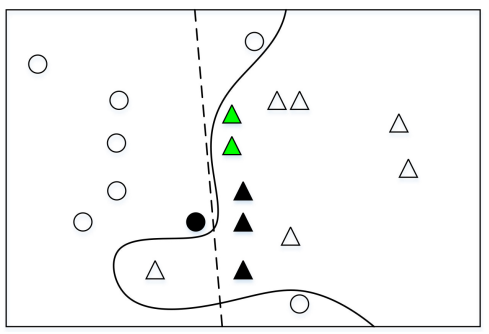

(b) Traditional entropy-based uncertainty sampling strategy.

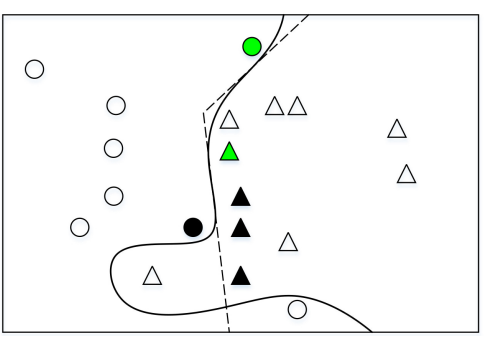

(c) NAASB sampling strategy.

Figure 3. Toy example to demonstrate the difference between traditional and NAASB sample selection process in active learning. Circles and triangles denote the instances from each class, solid circles and triangles denote original labeled instances, and the rest denote unlabeled instances. Green instances are selected at next iteration according to the sampling measurement. The solid lines denote the true decision boundary and the dashed lines denote the decision boundaries learned by classifiers based on the selected instances. Because the category of the instance is taken into consideration at sampling, NAASB can obtain a more precise decision boundary than traditional uncertainty sampling.

In this section, we present the NAASB active sampling framework in detail. Before describing our framework, it is necessary to state some basic assumptions: (i) during the learning process, the human labeler always tries his/her best for each labeling instance, is indefatigable (always answers the queries), and unique (only one) and (ii) the category of an image is definite, i.e., an image is assigned to a unique label.

\subsection{Overview of the NAASB sampling framework}

The scheme of the proposed active sampling strategy is summarized below:

1) Initialization: before the framework is fully workable, a bootstrap process is needed. During the first step, BoVW classifiers are trained with a few labeled data provided by a human annotator. The purpose of this process is to set up basic knowledge for classifiers. Initialization of the parameters is described in Section 3.2.2.

2) Selection: after the initialization is finished, the framework has generated basic (but not sufficient) knowledge for classification. In this stage, we aim to strengthen this knowledge by iterative learning. Details are shown in Section 3.2.1. Here, an SVM with a Gaussian $\chi^{2}$ kernel is chosen because it is the most efficient [53, 54]. The complete iterative process is divided into three steps: sample selection, annotation, and classifier training. At the beginning of each iteration, samples are first selected according to a certain measurement.

3) Annotation: selected samples are labeled by a human annotator, including steps for providing category information and moving samples into the corresponding categories of the labeled set.

4) Training: in the third stage of iteration, BoVW classifiers are trained with the newly generated labeled set. These iterations continue until the negative-accelerated criterion is met. The whole framework is described in Section 3.2.3.

5) Testing: in addition to traditional regular testing, a transfer testing strategy is introduced to fully evaluate the performance of the proposed active sampling framework. 


\subsection{Details of the NAASB framework}

\subsubsection{Measurement of NAASB}

In this section, the proposed active sampling framework is described in detail. Suppose we have an unlabeled set with each image belonging to one of $n$ possible classes. Active learning begins by measuring and choosing one or more images to be presented to the human annotator for correct labeling. The entropy based uncertainty measurement can be defined as [8]:

$$
D_{e}=-\sum_{i=1}^{n} P\left(y_{i} \mid z\right) \log P\left(y_{i} \mid z\right)
$$

where $P\left(y_{i} \mid z\right)$ is the estimated probability of sample $y_{i}$ given prior $z$. One deficiency of the traditional uncertainty metric described above is that it ignores the category distributions at the sampling stage [16], affecting the performance of sample selection and leading to the fact that selected samples need to be re-annotated [52]. To solve this problem, we introduce an certainty measurement calculated by BoVW into the traditional uncertainty-based sampling method to estimate the category distribution of samples. The class distribution measurements are estimated by

$$
\begin{aligned}
& c=\arg \max _{i=1}^{n} P_{i}^{A} \\
& D_{s}=\sqrt{\frac{1}{m} \sum_{i=1}^{m}\left(Y-Y_{i}^{c}\right)^{2}}
\end{aligned}
$$

where $P_{i}^{A}$ is the response of the classifier according to a certain $\mathrm{BoF}, c$ is the estimated category of the testing sample, $m$ is the number of samples in $c$, and $Y_{i}^{c}$ is the $i$-th labeled sample of $c$. Here, $D_{s}$ corresponds to the mean square error (MSE) [55] between the current sample and the samples of corresponding category in the labeled set. By taking the MSE of the labeled set into consideration, the representativeness is introduced into the model to improve the effectiveness of the proposed measurement.

The total measurement function proposed in this paper is given by

$$
D=\log \left(\left|\beta \operatorname{norm}\left(D_{e}\right)+(1-\beta) \operatorname{norm}\left(D_{s}\right)\right|\right)
$$

The uncertainty measurement $D_{e}$ provides an information metric for the samples, while the distribution of the classes is introduced by $D_{s}$. For each instance of $U$, we calculate both $D_{e}^{(j)}$ and $D_{s}^{(j)}$ measured by Eq. (2) and Eq. (3) respectively, and get vectors $\mathbf{D}_{\mathbf{E}}$ and $\mathbf{D}_{\mathbf{S}}$ given as $\mathbf{D}_{\mathbf{E}}=\left[D_{e}^{(1)}, D_{e}^{(2)}, \cdots, D_{e}^{(N)}\right]^{\mathbf{T}}$ and $\mathbf{D}_{\mathbf{S}}=\left[D_{s}^{(1)}, D_{s}^{(2)}, \cdots, D_{s}^{(N)}\right]^{\mathbf{T}}$, where $j \in\{1,2, \cdots, N\}$, and $N$ is the size of $U$. Operations of normalization are separately carried out on $\mathbf{D}_{\mathbf{E}}$ and $\mathbf{D}_{\mathbf{S}}$. Thus the normalized values $\operatorname{norm}\left(D_{e}^{(j)}\right)$ and $\operatorname{norm}\left(D_{s}^{(j)}\right)$ of the $j$-th instance are added to calculate the total measurement $D^{(j)}$.

The process of our pool-based sampling framework is given in detail in Fig. 4. Categories are represented by different marks. The process of sample selection and human annotation can be decomposed into six steps: i) Initialization: the BoVW classification module is trained with few samples labeled by human annotation. After initialization, the BoVW classifiers have a basic knowledge about the samples. ii) Entropy estimation: in unlabeled set $U$, the uncertainty based on entropy $D_{e}$ is calculated by Eq. (2) for each sample, which provides the basic metric for samples. (iii) Category estimation: categories of the selected samples are estimated by BoVW and the distribution of classes are calculated by Eq. (3). In this step, some samples are correctly categorized and others are wrongly categorized because of the performance of the BoVW classifier. iv) Human annotation: the human annotator checks the results of the BoVW classifier and corrects the misclassified samples. Samples with correct labels are then added into $L$. v) Retraining: the BoVW classifier is retrained by $L$ to improve its performance because the training samples now have correct labels and the selection of training samples corresponds to the distribution of samples set rather than a random selection. vi) Return to step (ii), and begin the next iteration until the process is convergent.

\subsubsection{Adaptive parameter setting}

As mentioned above, $\beta \in[0,1]$ is a decaying weight in Eq. (4), which satisfies

$$
\beta=k \mathrm{e}^{-w t}
$$




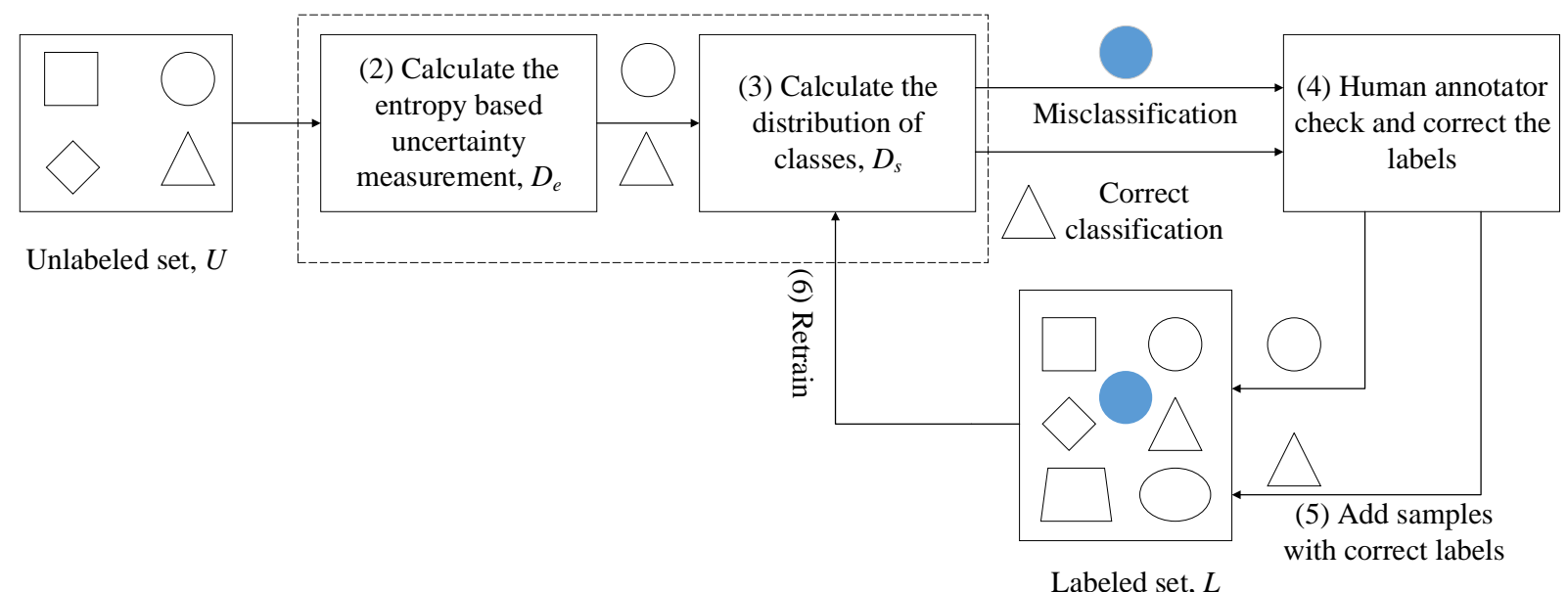

Figure 4. Toy example illustrating the working process of the proposed framework.

where $k$ is the initial value of $\beta, t$ is the number of iterations, and $w$ is set according to different categories. According to the learning curve of the negative-accelerated principle [50] mentioned above, we utilize a decaying weight because the more we train, the better the classifier may perform; thus, the BoVW metric module should be given a heavier weight to affect the total measurement. Moreover, because the scale, similarity, and quality of features between samples varies among categories, it is reasonable to set $\beta$ for each category individually, and the classification results are independent from $k$. For example, for two categories "car" and "sofa" containing 4 and 100 samples, respectively, the learning speed of classifiers for both classes will be different. It takes less time to train a classifier with fewer samples. Furthermore, the performance of the classifiers after training is affected by the number of training samples. The more samples a classifier has seen, the better it may perform. Last but not least, the features of a car are more stable than a sofa with less variation, and the similarity among samples for both categories are different. Thus, in this paper, instead of setting the same $\beta$ for each category, we utilize structural similarity (SSIM) [56] to reflect the relationships between samples in a category to dynamically initialize the value of $w$ for different categories of training sets. This improves the adaptivity of the sampling method to the variation of dataset. The similarity of $w$ between images $x$ and $y$ in the labeled set is given as follows:

$$
w=\frac{1}{N} \sum_{1 \leq x, y \leq N} \operatorname{SSIM}(x, y)=\frac{\left(2 \mu_{x} \mu_{y}+C_{1}\right)\left(2 \sigma_{x y}+C_{2}\right)}{\left(\mu_{x}^{2}+\mu_{y}^{2}+C_{1}\right)\left(\sigma_{x}^{2}+\sigma_{y}^{2}+C_{2}\right)}
$$

where $\mu_{x}$ and $\mu_{y}$ are the respective mean intensities of image $x$ and $y, \sigma_{x}$ and $\sigma_{y}$ are the standard deviations, and $\sigma_{x y}$ is the covariance. In addition, $C_{1}$ and $C_{2}$ are two constants to avoid instability when $\mu_{x}^{2}+\mu_{y}^{2}$ is very close to zero, and $N$ is the size of the category set.

\subsubsection{NAASB learning framework}

The proposed NAASB learning framework is presented in Algorithm 1. According to the learning principle, after the learning iterations are finished, the classifier is ready for classification tasks. Naturally, the negative-accelerated learning model provides a principle that determines when to stop the iteration process. For the practical application of computer vision, we simplified the measurement of negative-accelerated learning theory, and hence give the stop criterion as

$$
\Delta V_{n}=\alpha\left(V_{\max }-V_{\Sigma}\right) \leq V_{T}
$$

where $\Delta V_{i}$ describes the amount of increment in annotation quality after the $i$-th annotation, $\alpha \in(0,1]$ is the learning speed, $V_{\max }$ is the maximum performance a classifier may achieve, $V_{\Sigma}$ is the total quality achieved before the $n$-th iteration, $V_{\Sigma}<V_{\max }$, and $V_{T}$ is a relatively small value. Because the stop criterion is designed according to the 
negative-accelerated principle, $V_{\max }$ and $V_{\Sigma}$ are connected to the negative-accelerated learning principle by the above criterion. The iteration should stop when $\Delta V_{n}$ and $\Delta V_{n+1}$ are less than $V_{T}$.

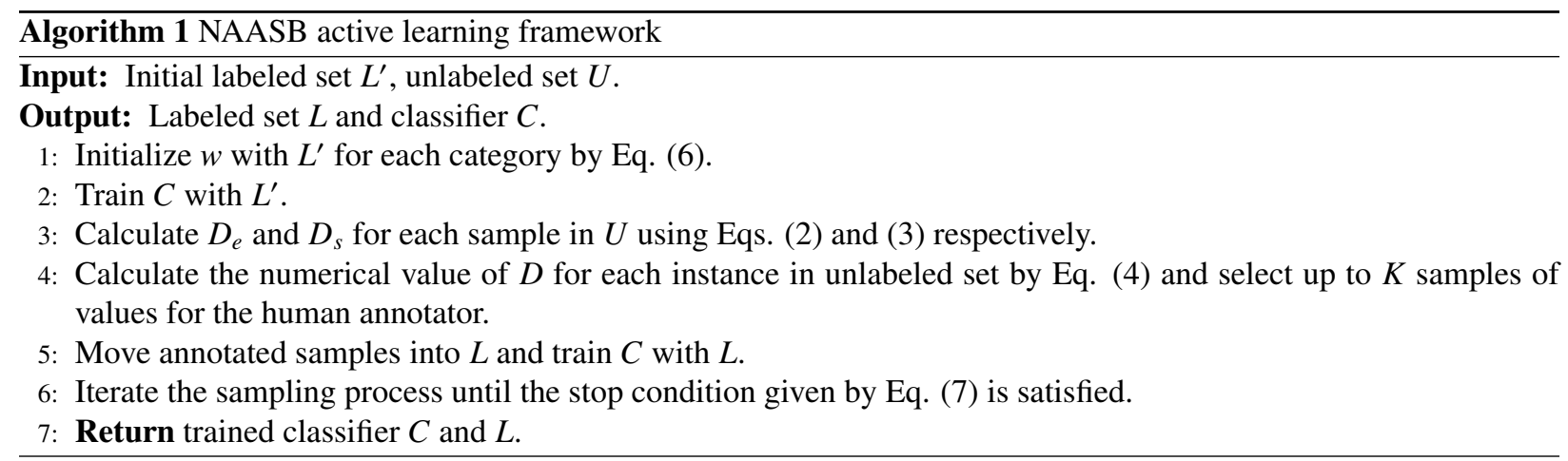

Compared with traditional active learning methods, the introduction of the negative-accelerated principle provides a simple but reasonable solution for when the training will be stopped based on the cognitive learning principle. Moreover, combining a BoVW module with the entropy measurement improves the quality of the sampling process. In return, the entropy-based uncertainty sampling strategy helps to improve the performance of the classifier, reducing the work of the human annotator. Thus, the whole framework is a bidirectional and self-improving scheme. In addition, the framework can lighten the burdensome annotation work for the human annotator by providing category distributions of samples to reduce the re-annotation cost.

In this paper, we also introduce the idea of transfer learning for evaluation, called transfer testing. Although the training and testing data are under same distribution, they are not from the same domain. In other words, they have the same categories, but the samples in these sets are not from the same dataset. Transfer testing is utilized because [39]: (i) there is limited availability of human labeled training data, which stays in the same feature space or has the same distribution, and cannot be guaranteed to be sufficient to avoid the over-fitting problem, and (ii) including related data in a different domain can expand the availability of the knowledge about the targeted future data.

As pointed out by [57], informativeness measures the ability of an instance to reduce the uncertainty of a model, whereas representativeness measures the degree to which an instance represents the overall input patterns of the unlabeled data. Here, the informativeness is measured by the uncertainty measured by entropy [8], and the representativeness is introduced into the proposed sampling strategy in the following ways. i) The classification module and parameters are initialized by a labeled set to bootstrap the model for the classifiers to generate basic (but not perfect) knowledge. ii) Samples are selected according to their informativeness and representativeness, and the selected samples are preliminarily annotated by the BoVW classifier, then sent to human annotator to ensure the samples are labeled correctly. iii) During the iterations, the classification module is retrained with newly generated labeled set to improve its performance and reduce the possibility of selecting outliers. With these features, the proposed NAASB sampling framework takes both informativeness and representativeness into consideration, and selects appropriate samples to improve the efficiency and effectiveness of training.

\section{Experiments and Analysis}

In this section, we first introduce the settings for our experiments, then present the experimental results and analyses of NAASB compared with traditional and state-of-the-art active learning methods [8, 58, 59, 49, 60], which are the most representative approaches in this field. The features of each method are listed in Table 1. According to previous studies [1, 2], in traditional sampling strategies, the sampling and training processes are separated in image classification tasks, which means the image classifier is trained after sample selection is finished. Thus, in the experiment, both separated strategies and unified methods are compared to fully evaluate the performance. The settings of experiments are described in Section 4.1. Comparative results and analysis are given in Section 4.2. 
Table 1. Features of compared strategies and methods

\begin{tabular}{cc}
\hline Strategy/Method & Features \\
\hline Holub [8] & Separated sampling \& classification \\
Li [49] & Unified sampling \& classification \\
Kanamori [58] & Separated sampling \& classification \\
Joshi [59] & Unified sampling \& classification \\
Yu [60] & Separated sampling \& classification \\
\hline
\end{tabular}

\subsection{Data and settings}

The tests are divided into three parts: the regular test, integrated transfer test, and regressive transfer test. The regular test was performed using the MSRC dataset [61]. In this test, 16 categories were selected that contain at least 15 training and 15 testing images for each category. A set of 40 fully-annotated images were chosen from the training set such that each object category had at least two instances to initialize the model parameters. After the regular test, the trained classifier was utilized for the transfer test. Here, we divided the transfer test into two parts: the integrated test and regressive transfer test. In the integrated transfer test, the testing data is composed of different datasets, covering all categories of the original training dataset. Samples of common categories are selected from both PASCAL VOC 2007 and Caltech-101, and their results are respectively shown to give the details of classification. We proposed the NAASB sampling framework to solve the problem of selecting samples with one label, and it is more effective in the field of traditional active learning. To compare with other one label active learning methods proposed in $[8,49,58,60]$, images with more than one label are discarded for the PASCAL VOC 2007 dataset, which is very popular in computer vision community. In the regressive transfer test, the testing data was taken from the same dataset, covering part of the original training categories. Introducing both tests provides a complete evaluation of the learning strategies. The integrated transfer test was conducted on a hybrid dataset that included a part of the PASCAL VOC 2007 [62] and Caltech-101 [63]. For those categories that do not appear in both datasets, images were collected from Google to build the categories. Common semantic categories were selected to ensure the training and testing data had the same semantics distribution. Graz-02 [64] was used for the regressive transfer test to further evaluate the performance. In particular, some samples that can be classified into multiple categories were selected from the "Bicycle" and "Car" categories to form a new category, "House," to cover all datasets in the integrated transfer test. A one-versus-all strategy was utilized to train the SVM classifiers. All categories selected for the tests are given in Table 2. Sample images are shown in Fig. 5. Here, we set the perceptive parameters according to previous research [50]: $V_{\max }=100 \%, \alpha=0.05, k=0.8$, and $K=5 \%$. Settings for the BoVW parameters were also chosen according to previous research [54]. The size of the vocabulary was 1000, SIFTs [65] and linear SVMs were respectively used as the feature descriptor and classifier. Optimized settings were used for the other methods.

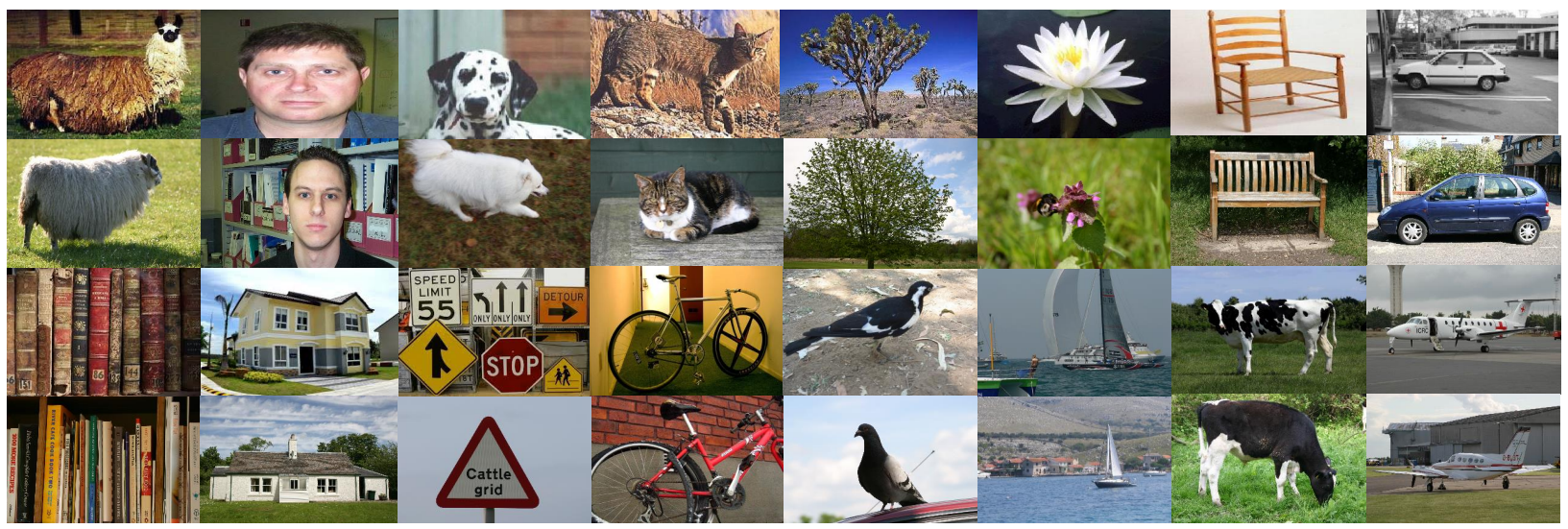

Figure 5. Samples from all datasets. 
Table 2. Constitution of datasets for training and testing.

\begin{tabular}{ccccc}
\hline Training & \multicolumn{5}{c}{ Testing } \\
\hline MSRC & PASCAL VOC 2007 & Caltech-101 & Graz-02 & Google Image \\
\hline Sheep & $\sqrt{ }$ & $\sqrt{ }$ & & \\
Human & $\sqrt{ }$ & $\sqrt{ }$ & $\sqrt{ }$ & \\
Dog & $\sqrt{ }$ & $\sqrt{ }$ & & \\
Cat & $\sqrt{ }$ & $\sqrt{ }$ & & \\
Tree & & $\sqrt{ }$ & & \\
Flower & & $\sqrt{ }$ & & \\
Chair & $\sqrt{ }$ & $\sqrt{ }$ & $\sqrt{ }$ & \\
Car & $\sqrt{ }$ & $\sqrt{ }$ & & $\sqrt{ }$ \\
Sign & & & $\sqrt{ }$ & $\sqrt{ }$ \\
House & & & & $\sqrt{ }$ \\
Book & & & & \\
Plane & $\sqrt{ }$ & & & \\
Cow & $\sqrt{ }$ & & $\sqrt{ }$ \\
Boat & $\sqrt{ }$ & & & \\
Bicycle & $\sqrt{ }$ & & & \\
Bird & $\sqrt{ }$ & & & \\
\hline
\end{tabular}

\subsection{Results and analysis}

The experimental results are composed of four parts: i) evaluation of parameter $V_{T}$ in Eq. (7), ii) comparison of the classification performance of different sampling strategies on the selected datasets, iii) detailed classification results presented by confusion matrices, and iv) effectiveness of the reduction of additional annotation work.

The overall experimental results are presented in Figs. 6 and 7. Fig. 6 (a) shows the relationships between the value of $\beta \mathrm{s}$ and number of iterations for the categories of MSRC. The strategy of using fixed $\beta$ for all categories is represented by a line. We can see from the figure that every category in the training set has unique characteristics. Thus, we can obtain various $w$ s for training samples. Setting a fixed $\beta$ for each category cannot sufficiently reflect its inner properties. Here, by utilizing the SSIM measurement, the inner similarities of the categories are measured to reflect the characteristics of the samples. Thus, it is reasonable to initialize every $w$ separately.

Fig. 6 (b) shows the overall performance of all 16 categories on the MSRC dataset. We can see from these results that the performance of the active learning framework using the NAASB sampling strategy outperforms other strategies and achieves better performance on most tests. In particular, NAASB with variable $\beta$ for each category achieves better performance than other strategies. This is because separately setting model parameters for categories according to their circumstances is helpful for selecting higher quality samples so that the classifier can improve its performance. As iterations progress, the difference in performance between NAASB and the traditional entropy-based and random sampling strategy increases.

Fig. 6 (c) shows the performance of NAASB for different $V_{T}$ s. According to the stopping criterion, we tested the value of $V_{T}$ from $1 \%$ to $5 \%$ in intervals of $1 \%$. Values of $V_{T}$ were chosen according to the corresponding performance. We can see that $V_{T}=2 \%$ achieves the best performance. For $V_{T}=1 \%$, the performance grows so slowly that the iteration process stops early. For $V_{T}=3 \%$, performance is better than that of $V_{T}=2 \%$ at first. However, although the training process converges, the increment oscillates, affecting the final precision. For $V_{T}=4 \%$ and $V_{T}=5 \%$, in the early stages of iteration, the performance grows relatively fast; however, because of the excessive value of $V_{T}$, the learning process suffers from over-fitting because the convergence criterion is too loose. After some iterations, the performance starts to decline. In contrast to the results using $V_{T}=2 \%$, the training process converges at low performance. Here, $V_{T}$ works like a valve between the core components and pool of samples. If the value of $V_{T}$ is too small, the learning process will converge at an early stage before the framework obtains better performance and the better samples have been selected. Nevertheless, if $V_{T}$ is larger than proper value, the final performance is affected by the oscillation during the learning process, which means that samples of both high and normal qualities are selected. In contrast, if $V_{T}$ is too large, although the process will still converge, the framework will select more mediocre samples 
instead of high quality ones, the performance of classifier will raise fleetingly, and then drop quickly.

Fig. 6 (d) shows the variation of average MSE between selected samples and the labeled set on MSRC for each iteration. In general, the value of MSE decreases as the iterations progress. At the same time, the accuracy of the classifier increases and the number of human labeled samples decreases, indicating that the proposed sampling framework is selecting more correlative samples from the unlabeled set.

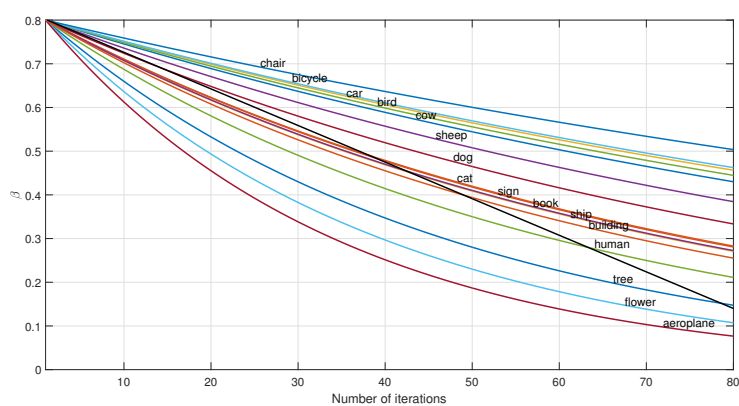

(a) Value of $\beta$ s for each category of MSRC.

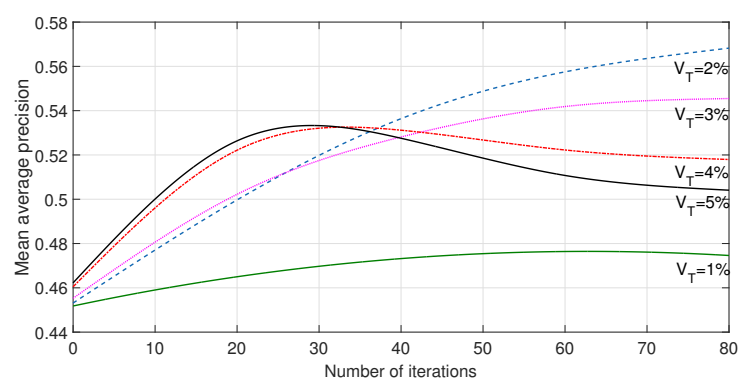

(c) Classification results of MSRC with different values of $V_{T}$.

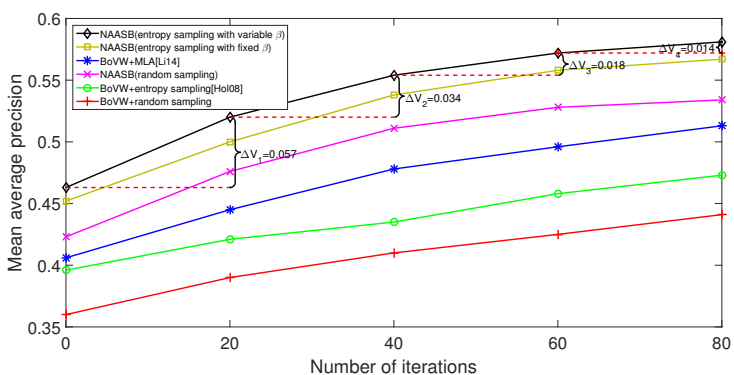

(b) Results of active sampling \& regular testing on MSRC.

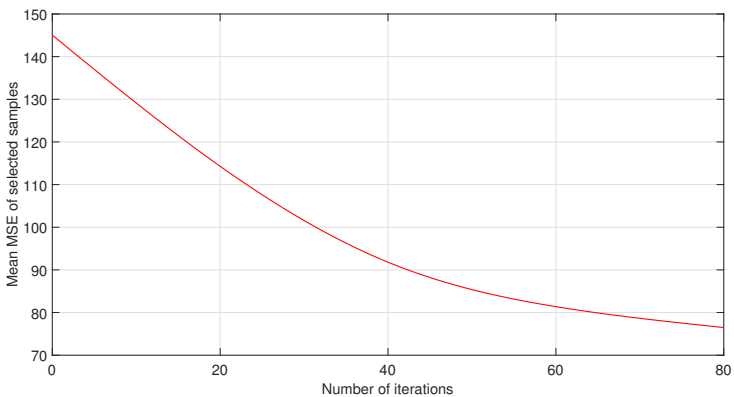

(d) Variation of average MSE between selected samples and the labeled set.

Figure 6. Experimental results of regular testing.

Figs. 7 (a)-(e) give the results of the integrated transfer tests on Caltech-101, PASCAL VOC 2007 and Google Images. Results of the regressive transfer test on Graz-02 are given in Fig. 7(f). We can conclude that introducing certainty measurement into the traditional entropy-based strategy makes the sampling process more effective. Specifically, compared with strategies that only consider entropy measurement, random sampling with NAASB achieves better performance. This proves that taking category distribution into consideration is effective, which is consistent with existing research [52]. For the testing results on common categories of Caltech-101 and PASCAL VOC 2007, although the performances in Figs. 7 (b) and 7 (d) vary from the category settings in Table 2, the trend is similar, indicating that there is no bias in the sample selection from any dataset because the testing data from both categories are equally strange to the classifier. The performance differences are due to the features of each dataset. For most common categories in PASCAL VOC 2007, the samples are more outstanding and visually similar, thus it is much easier to generate a visual vocabulary of higher quality, which is beneficial for classification. At the same time, for the transfer test, how to efficaciously transfer knowledge from different datasets is a remaining problem. We cannot ignore the fact that the performance of the active sampling strategies are not satisfactory over some categories such as "cat," "dog," and "bird." This is because most samples are not salient enough and filled with different numbers of noisy objects, which adversely affect the BoVW-based classifier's performance. In contrast, the quality of most categories from PASCAL VOC 2007 and Google Images is relatively high with outstanding and centered objects and is helpful for narrowing the semantic gap [66] and generating a more compact visual vocabulary [67], making the classification much more precise. Even so, we can see broad potential of the proposed active sampling framework. If properly combined with other image processing techniques such as segmentation [29] to improve both the quality of the visual words of the BoVWs and testing samples, better performance could be achieved on the difficult categories. 
Moreover, introducing the techniques of transfer learning methods is also a great help.

Fig. 8 displays the confusion table for all tests, which shows the average classification performances for individual categories of the dataset and how the different categories are confused. Confusion occurs when the images contains similar scenes and backgrounds, e.g., "dog" vs. "cow," "cat" vs. "cow," or "dog” vs. "cat." Only rates higher than 5\% are shown in the figure for clarity.
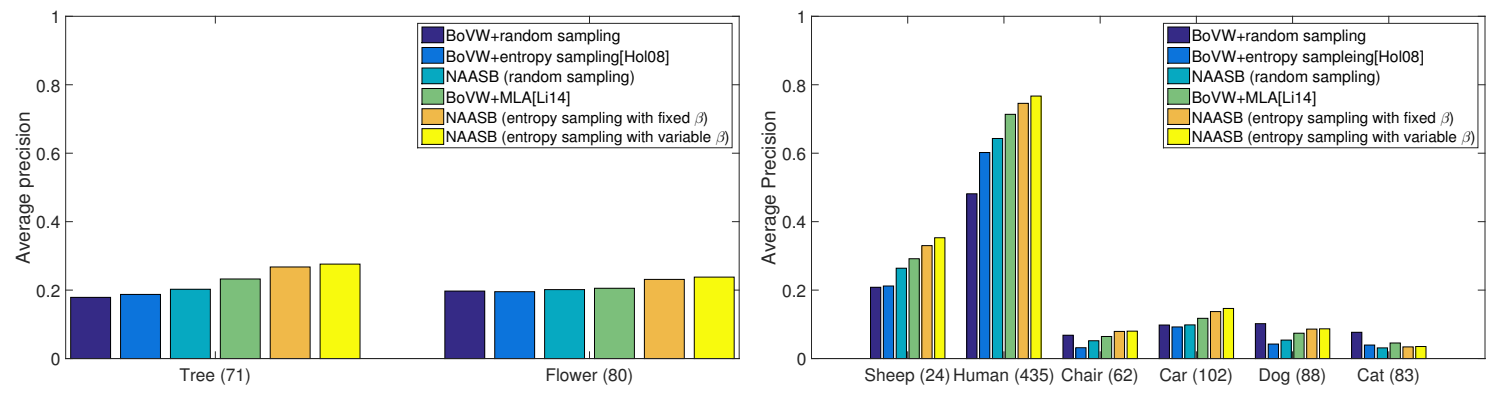

(a) Results of active sampling \& integrated transfer testing on featured categories from Caltech-101.

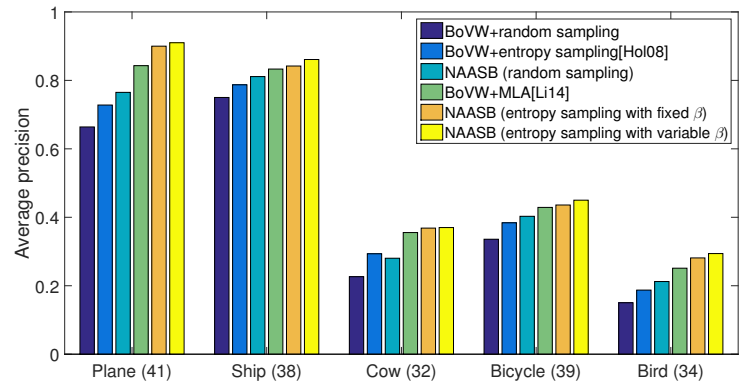

(b) Results of active sampling \& integrated transfer testing on common categories from Caltech-101.

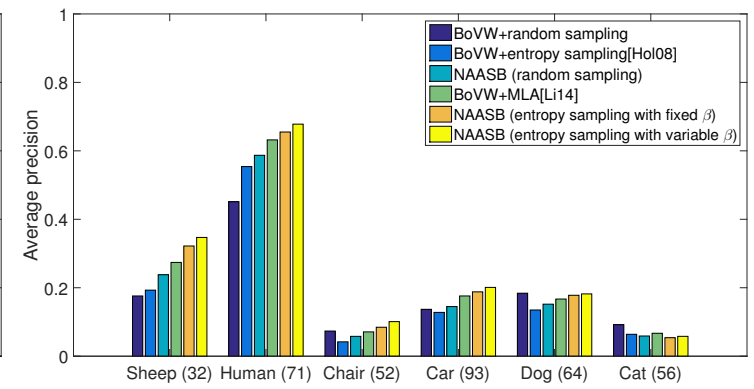

(c) Results of active sampling \& integrated transfer testing on featured categories from PASCAL VOC 2007.

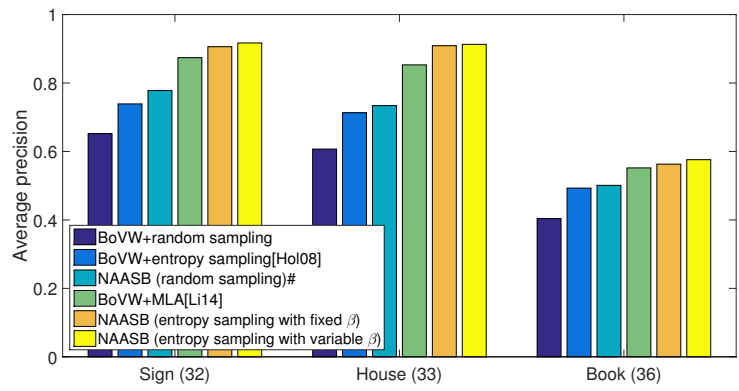

(e) Results of active sampling \& integrated transfer testing on featured categories from Google Image. (d) Results of active sampling \& integrated transfer testing on common categories from PASCAL VOC 2007.

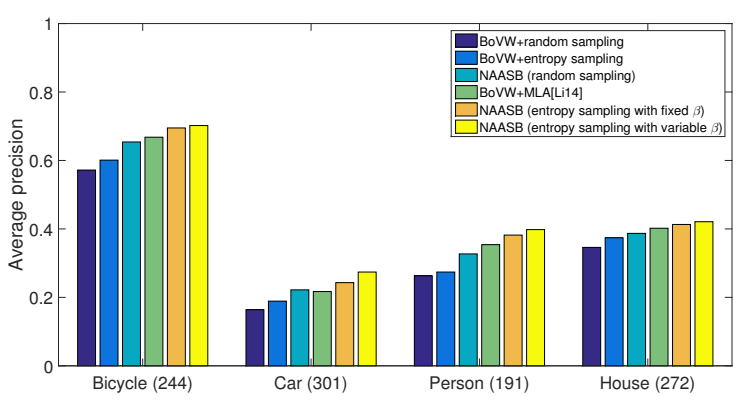

(f) Results of regressive transfer testing on Graz-02.

Figure 7. Experimental results of transfer testing.

Furthermore, comparative results of the relevant methods $[58,59,60]$ are given in Table 3. From the results, we can see that compared with other methods, the proposed NAASB framework, which takes category information into consideration, achieves better performance with respect to mean average precision (MAP), reflecting the effectiveness of incorporating category information. On most categories, substantial improvement is observed.

Last but equally important, to clearly reflect whether the proposed NAASB sampling framework can reduce additional annotation work required of a human annotator, we respectively show the number of selected and re-annotated samples for the MSRC dataset after a certain number of iterations in Table 4. Performance is measured by MAP. The needed re-annotations for the other datasets can be roughly measured by the average precision given in Fig. 7. At the 


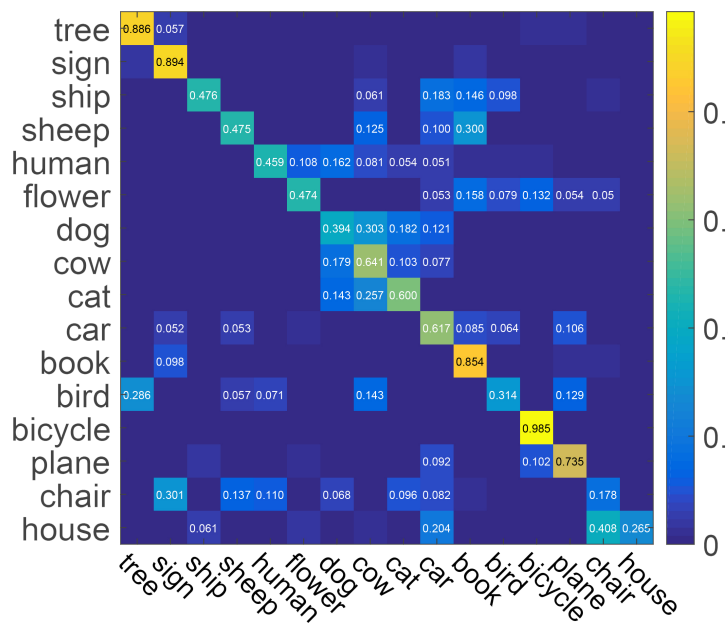

(a) Result of regular testing on MSRC.

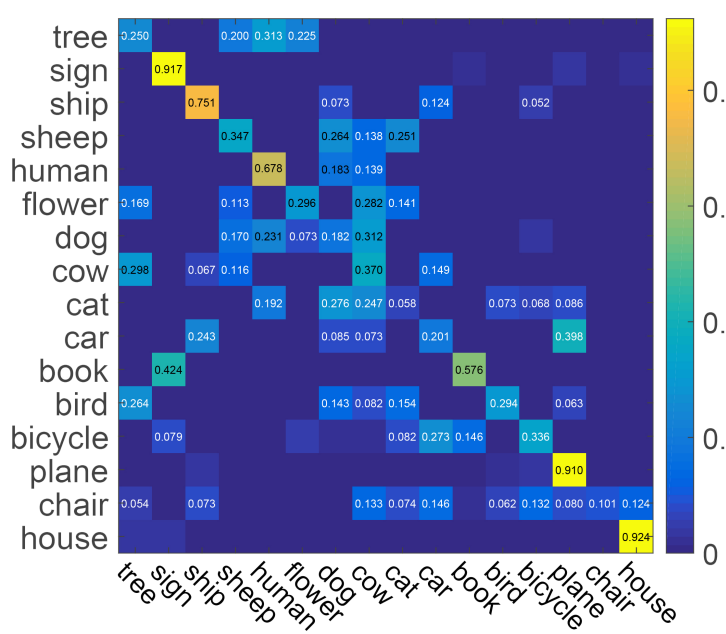

(c) Result of integrated transfer testing. Samples of common categories are selected from PASCAL VOC 2007.

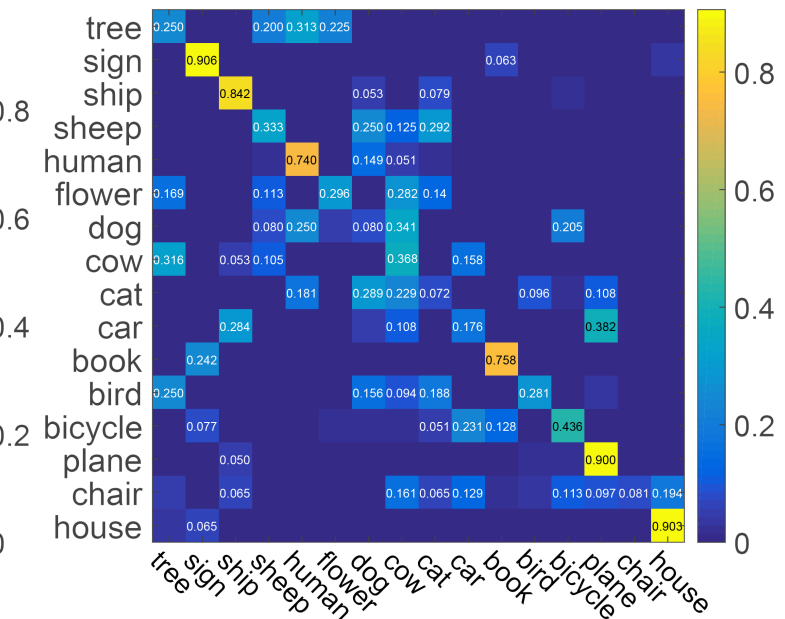

(b) Result of integrated transfer testing. Samples of common categories are selected from Caltech-101.

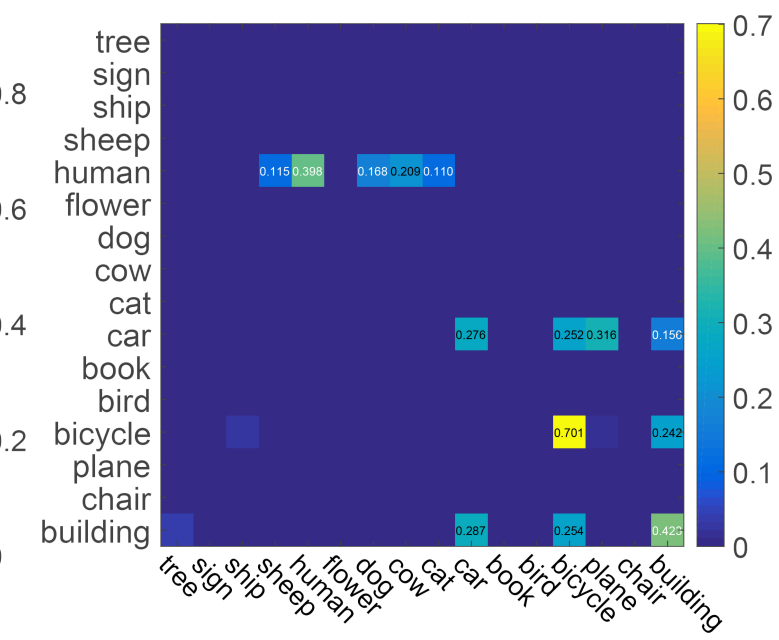

(d) Result of regressive transfer testing on Graz-02.

Figure 8. Confusion matrices for all tests. 
Table 3. Comparative results of the proposed framework and other label-wise active learning methods in transfer testing. Methods with the best performance for each category are marked by asterisks.

\begin{tabular}{ccccc}
\hline & Kanamori [58] & Joshi [59] & Yu [60] & NAASB \\
\hline Sheep & 0.249 & 0.296 & 0.320 & $0.340^{*}$ \\
Human & 0.557 & 0.639 & 0.682 & $0.709^{*}$ \\
Dog & 0.094 & 0.117 & $0.160^{*}$ & 0.131 \\
Cat & 0.029 & 0.066 & 0.056 & $0.065^{*}$ \\
Tree & 0.172 & 0.203 & 0.237 & $0.250^{*}$ \\
Flower & 0.164 & 0.217 & 0.269 & $0.298^{*}$ \\
Chair & 0.045 & 0.079 & $0.121^{*}$ & 0.091 \\
Car & 0.056 & 0.086 & 0.129 & $0.189^{*}$ \\
Sign & 0.742 & 0.811 & 0.879 & $0.906^{*}$ \\
House & 0.763 & 0.852 & 0.887 & $0.903^{*}$ \\
Book & 0.464 & 0.572 & 0.696 & $0.758^{*}$ \\
Plane & 0.703 & 0.824 & 0.881 & $0.910^{*}$ \\
Cow & 0.264 & 0.317 & 0.332 & $0.370^{*}$ \\
Ship & 0.694 & 0.742 & $0.807^{*}$ & 0.751 \\
Bicycle & 0.307 & 0.354 & 0.363 & $0.336^{*}$ \\
Bird & 0.238 & 0.274 & $0.323^{*}$ & 0.294 \\
\hline MAP & 0.346 & 0.403 & 0.446 & $\mathbf{0 . 4 6 4}$ \\
\hline
\end{tabular}

end of each iteration, selected samples need to be moved into the training sets of the corresponding categories, thus the better the BoF based classifier performs, the less work needs to be done for re-annotation. We can see from the results that the ratio of re-annotated samples decreases as performance improves, proving that the NAASB sampling framework can effectively reduce the additional annotation work required of a human annotator. Before the iterations, the classifier is initialized to generate basic (but not sufficient) knowledge for classification after the initialization step mentioned in Algorithm 1. Parameter $V_{\Sigma}$ is $46.1 \%$ instead of zero on the training data from MSRC, indicating that the iteration will not stop after only one iteration.

Table 4. Relationships between annotation work and the performance of NAASB on MSRC.

\begin{tabular}{ccc}
\hline Number of iterations & Performance of classifier & Percentage of re-annotated samples \\
\hline 0 & $46.1 \%$ & $75.4 \%$ \\
20 & $50 \%$ & $63.2 \%$ \\
40 & $53.8 \%$ & $58.3 \%$ \\
60 & $55.8 \%$ & $57.1 \%$ \\
80 & $56.7 \%$ & $50 \%$ \\
\hline
\end{tabular}

The experimental results of the proposed framework with different classifiers are given in Table 5. We can see that with advanced classifiers, better classification accuracy can be achieved, indicating the fact that our framework does not dependent on specific classifiers. This is because we target and propose a novel sample selection strategy that is classifier independent in this paper instead of improving the classifier itself for image classification tasks.

Table 5. Performance of NAASB with different classifiers on MSRC.

\begin{tabular}{cccccc}
\hline Number of iterations & 0 & 20 & 40 & 60 & 80 \\
\hline BoVW & $46.1 \%$ & $50 \%$ & $53.8 \%$ & $55.8 \%$ & $56.7 \%$ \\
SPM [30] & $49.6 \%$ & $55.9 \%$ & $62.4 \%$ & $64.3 \%$ & $65.6 \%$ \\
WSA [67] & $52.8 \%$ & $58.1 \%$ & $64.3 \%$ & $65.9 \%$ & $66.7 \%$ \\
\hline
\end{tabular}




\section{Conclusion}

In this paper, an entropy-based sampling framework called NAASB was proposed for active sampling in image classification by investigating the well-known psychological Rescorla-Wagner model and image classification method - A new measurement function was presented for the traditional entropy-based sampling strategy. In addition, an adaptive initialization method for the parameter was presented. Experiments on multiple real-world datasets show the effectiveness of our framework. For the challenge of image classification, we believe that the active learning framework will improve classification performance and reduce the duplication of human labor to reduce the annotation cost. Future work will investigate both the inner and outer measurements of categories to further improve the accuracy.

\section{Acknowledgments}

This research was supported by the National Science Foundation of China (Grant Nos. 61171184 and 61201309).

\section{References}

[1] D. Angluin, Queries and concept learning, Machine Learning 2 (4) (1988) 319-342. doi:10.1007/BF00116828. URL http://dx.doi.org/10.1007/BF00116828

[2] D. Cohn, L. Atlas, R. Ladner, Improving generalization with active learning, Machine Learning 15 (2) (1994) $201-221$. doi:10.1007/BF00993277.

URL http://dx.doi.org/10.1007/BF00993277

[3] D. D. Lewis, J. Catlett, Heterogenous uncertainty sampling for supervised learning., in: ICML, Vol. 94, 1994, pp. 148-156.

[4] A. T. Da Silva, A. X. Falcão, L. P. Magalhães, Active learning paradigms for cbir systems based on optimum-path forest classification, Pattern Recognition 44 (12) (2011) 2971-2978.

[5] F. Sun, Y. Xu, J. Zhou, Active learning svm with regularization path for image classification, Multimedia Tools and Applications (2014) 1-16.

[6] Y. Fu, X. Zhu, B. Li, A survey on instance selection for active learning, Knowledge and Information Systems 35 (2) (2013) $249-283$.

[7] B. Settles, M. Craven, An analysis of active learning strategies for sequence labeling tasks, in: Proceedings of the Conference on Empirical Methods in Natural Language Processing, Association for Computational Linguistics, 2008, pp. 1070-1079.

[8] A. Holub, P. Perona, M. C. Burl, Entropy-based active learning for object recognition, in: Computer Vision and Pattern Recognition Workshops, 2008. CVPRW'08. IEEE Computer Society Conference on, IEEE, 2008, pp. 1-8.

[9] N. A. H. Mamitsuka, Query learning strategies using boosting and bagging, in: Machine Learning: Proceedings of the Fifteenth International Conference (ICML'98), Morgan Kaufmann Pub, 1998, p. 1.

[10] A. K. McCallumzy, K. Nigamy, Employing em and pool-based active learning for text classification, in: Machine Learning: Proceedings of the Fifteenth International Conference, ICML, Citeseer, 1998.

[11] T. Gao, D. Koller, Active classification based on value of classifier, in: Advances in Neural Information Processing Systems, 2011, pp. 1062-1070.

[12] B. Settles, M. Craven, S. Ray, Multiple-instance active learning, in: Advances in neural information processing systems, 2008, pp. $1289-1296$.

[13] Y. Guo, R. Greiner, Optimistic active-learning using mutual information., in: IJCAI, Vol. 7, 2007, pp. 823-829.

[14] S. Geman, E. Bienenstock, R. Doursat, Neural networks and the bias/variance dilemma, Neural computation 4 (1) (1992) 1-58.

[15] D. D. Lewis, W. A. Gale, A sequential algorithm for training text classifiers, in: Proceedings of the 17th annual international ACM SIGIR conference on Research and development in information retrieval, Springer-Verlag New York, Inc., 1994, pp. 3-12.

[16] Y. Fu, X. Zhu, B. Li, A survey on instance selection for active learning, Knowledge and information systems 35 (2) (2013) $249-283$.

[17] S. Savarese, J. Winn, A. Criminisi, Reducing labeling effort for stuctured prediction tasks, in: Proceedings of the 20th national conference on artificial intelligence (AAAI), 2005, pp. 746-751.

[18] R. Gilad-Bachrach, A. Navot, N. Tishby, Margin based feature selection - theory and algorithms, in: Proceedings of the Twenty-first International Conference on Machine Learning, ICML '04, ACM, New York, NY, USA, 2004, pp. 43-. doi:10.1145/1015330.1015352. URL http://doi . acm.org/10.1145/1015330.1015352

[19] M. Lindenbaum, S. Markovitch, D. Rusakov, Selective sampling for nearest neighbor classifiers, Machine learning 54 (2) (2004) $125-152$.

[20] J. Qin, N. H. Yung, Scene categorization via contextual visual words, Pattern Recognition 43 (5) (2010) $1874-1888$. doi:http://dx.doi.org/10.1016/j.patcog.2009.11.009. URL http://www.sciencedirect.com/science/article/pii/S0031320309004245

[21] N. M. Elfiky, F. S. Khan, J. van de Weijer, J. Gonzlez, Discriminative compact pyramids for object and scene recognition, Pattern Recognition 45 (4) (2012) 1627 - 1636. doi:http://dx.doi.org/10.1016/j.patcog.2011.09.020. URL http://www.sciencedirect.com/science/article/pii/S0031320311003992

[22] F. Wang, Y.-G. Jiang, C.-W. Ngo, Video event detection using motion relativity and visual relatedness, in: Proceedings of the 16th ACM International Conference on Multimedia, MM '08, ACM, New York, NY, USA, 2008, pp. 239-248. doi:10.1145/1459359.1459392. URL http://doi . acm.org/10.1145/1459359.1459392

[23] J. Liu, Y. Yang, I. Saleemi, M. Shah, Learning semantic features for action recognition via diffusion maps, Computer Vision and Image Understanding 116 (3) (2012) 361 - 377, special issue on Semantic Understanding of Human Behaviors in Image Sequences. doi:http://dx.doi.org/10.1016/j.cviu.2011.08.010.

URL http://www.sciencedirect.com/science/article/pii/S1077314211002116 
[24] G. Csurka, C. Dance, L. Fan, J. Willamowski, C. Bray, Visual categorization with bags of keypoints, in: Workshop on statistical learning in computer vision, ECCV, Vol. 1, 2004, pp. 1-2.

[25] J. Yang, Y.-G. Jiang, A. G. Hauptmann, C.-W. Ngo, Evaluating bag-of-visual-words representations in scene classification, in: Proceedings of the international workshop on Workshop on multimedia information retrieval, ACM, 2007, pp. 197-206.

[26] J. Krapac, J. Verbeek, F. Jurie, Modeling spatial layout with fisher vectors for image categorization, in: Computer Vision (ICCV), 2011 IEEE International Conference on, IEEE, 2011, pp. 1487-1494.

[27] J. Yuan, Y. Wu, M. Yang, Discovery of collocation patterns: from visual words to visual phrases, in: Computer Vision and Pattern Recognition, 2007. CVPR'07. IEEE Conference on, IEEE, 2007, pp. 1-8.

[28] S.-W. Choi, C. H. Lee, I. K. Park, Scene classification via hypergraph-based semantic attributes subnetworks identification, in: Computer Vision-ECCV 2014, Springer, 2014, pp. 361-376.

[29] Y. Chai, E. Rahtu, V. Lempitsky, L. Van Gool, A. Zisserman, Tricos: A tri-level class-discriminative co-segmentation method for image classification, in: Computer Vision-ECCV 2012, Springer, 2012, pp. 794-807.

[30] S. Lazebnik, C. Schmid, J. Ponce, Beyond bags of features: Spatial pyramid matching for recognizing natural scene categories, in: Computer Vision and Pattern Recognition, 2006 IEEE Computer Society Conference on, Vol. 2, IEEE, 2006, pp. $2169-2178$.

[31] J. Wang, J. Yang, K. Yu, F. Lv, T. Huang, Y. Gong, Locality-constrained linear coding for image classification, in: Computer Vision and Pattern Recognition (CVPR), 2010 IEEE Conference on, IEEE, 2010, pp. 3360-3367.

[32] J. C. van Gemert, C. J. Veenman, A. W. Smeulders, J.-M. Geusebroek, Visual word ambiguity, Pattern Analysis and Machine Intelligence, IEEE Transactions on 32 (7) (2010) 1271-1283.

[33] H. Bannour, C. Hudelot, Hierarchical image annotation using semantic hierarchies, in: Proceedings of the 21 st ACM international conference on Information and knowledge management, ACM, 2012, pp. 2431-2434.

[34] Y. Lu, L. Zhang, J. Liu, Q. Tian, Constructing concept lexica with small semantic gaps, Multimedia, IEEE Transactions on 12 (4) (2010) 288-299.

[35] J. Deng, A. C. Berg, L. Fei-Fei, Hierarchical semantic indexing for large scale image retrieval, in: Computer Vision and Pattern Recognition (CVPR), 2011 IEEE Conference on, IEEE, 2011, pp. 785-792.

[36] L. Wu, S. C. Hoi, N. Yu, Semantics-preserving bag-of-words models and applications, Image Processing, IEEE Transactions on 19 (7) (2010) $1908-1920$.

[37] J. Deng, A. Berg, K. Li, L. Fei-Fei, What does classifying more than 10,000 image categories tell us?, in: K. Daniilidis, P. Maragos, N. Paragios (Eds.), Computer Vision ECCV 2010, Vol. 6315 of Lecture Notes in Computer Science, Springer Berlin Heidelberg, 2010, pp. 71-84.

[38] W. Dai, Q. Yang, G.-R. Xue, Y. Yu, Boosting for transfer learning, in: Proceedings of the 24th international conference on Machine learning, ACM, 2007, pp. 193-200.

[39] L. Shao, F. Zhu, X. Li, Transfer learning for visual categorization: A survey, Neural Networks and Learning Systems, IEEE Transactions on PP (99) (2014) 1-16.

[40] M. Palatucci, D. Pomerleau, G. E. Hinton, T. M. Mitchell, Zero-shot learning with semantic output codes, in: Advances in neural information processing systems, 2009, pp. 1410-1418.

[41] L. Fei-Fei, R. Fergus, P. Perona, One-shot learning of object categories, Pattern Analysis and Machine Intelligence, IEEE Transactions on 28 (4) (2006) 594-611.

[42] P. Kankuekul, A. Kawewong, S. Tangruamsub, O. Hasegawa, Online incremental attribute-based zero-shot learning, in: Computer Vision and Pattern Recognition (CVPR), 2012 IEEE Conference on, IEEE, 2012, pp. 3657-3664.

[43] X. Glorot, A. Bordes, Y. Bengio, Domain adaptation for large-scale sentiment classification: A deep learning approach, in: Proceedings of the 28th International Conference on Machine Learning (ICML-11), 2011, pp. 513-520.

[44] J. Jiang, C. Zhai, Instance weighting for domain adaptation in nlp, in: ACL, Vol. 7, 2007, pp. 264-271.

[45] K. Huang, D. Tao, Y. Yuan, X. Li, T. Tan, Biologically inspired features for scene classification in video surveillance, Systems, Man, and Cybernetics, Part B: Cybernetics, IEEE Transactions on 41 (1) (2011) 307-313.

[46] Q. Tang, N. Sang, H. Liu, Learning to detect contours in natural images via biologically motivated schemes, in: Image Processing (ICIP), 2013 20th IEEE International Conference on, 2013, pp. 123-126.

[47] T. Tang, H. Qiao, Improving invariance in visual classification with biologically inspired mechanism, Neurocomputing 133 (2014) 328-341.

[48] P. Donmez, J. G. Carbonell, J. Schneider, Efficiently learning the accuracy of labeling sources for selective sampling, in: Proceedings of the 15th ACM SIGKDD international conference on Knowledge discovery and data mining, ACM, 2009, pp. $259-268$.

[49] X. Li, Y. Guo, Multi-level adaptive active learning for scene classification, in: Computer Vision-ECCV 2014, Springer, 2014 , pp. $234-249$.

[50] R. A. Rescorla, A. R. Wagner, et al., A theory of pavlovian conditioning: Variations in the effectiveness of reinforcement and nonreinforcement, Classical conditioning II: Current research and theory 2 (1972) 64-99.

[51] S. Siegel, L. G. Allan, The widespread influence of the rescorla-wagner model, Psychonomic Bulletin \& Review 3 (3) (1996) 314-321.

[52] W. Wu, Y. Liu, M. Guo, Constructing training distribution by minimizing variance of risk criterion for visual category learning, in: Image Processing (ICIP), 2012 19th IEEE International Conference on, IEEE, 2012, pp. 101-104.

[53] P. H. Gosselin, M. Cord, A comparison of active classification methods for content-based image retrieval, in: Proceedings of the 1st international workshop on Computer vision meets databases, ACM, 2004, pp. 51-58.

[54] Y.-G. Jiang, C.-W. Ngo, J. Yang, Towards optimal bag-of-features for object categorization and semantic video retrieval, in: Proceedings of the 6th ACM international conference on Image and video retrieval, ACM, 2007, pp. 494-501.

[55] R. Dosselmann, X. Yang, A comprehensive assessment of the structural similarity index, Signal, Image and Video Processing 5 (1) (2011) 81-91. doi:10.1007/s11760-009-0144-1.

URL http://dx.doi.org/10.1007/s11760-009-0144-1

[56] Z. Wang, A. Bovik, H. Sheikh, E. Simoncelli, Image quality assessment: from error visibility to structural similarity, Image Processing, IEEE Transactions on 13 (4) (2004) 600-612. doi:10.1109/TIP.2003.819861.

[57] S. jun Huang, R. Jin, Z. hua Zhou, Active learning by querying informative and representative examples, in: J. Lafferty, C. Williams, 
J. Shawe-Taylor, R. Zemel, A. Culotta (Eds.), Advances in Neural Information Processing Systems 23, Curran Associates, Inc., 2010 , pp. 892-900.

URL http://papers.nips.cc/paper/4176-active-learning-by-querying-informative-and-representative-examples.pdf

[58] T. Kanamori, Pool-based active learning with optimal sampling distribution and its information geometrical interpretation, Neurocomputing 71 (13) (2007) 353 - 362, dedicated Hardware Architectures for Intelligent SystemsAdvances on Neural Networks for Speech and Audio Processing. doi:http://dx.doi.org/10.1016/j.neucom.2006.11.024.

URL http://www.sciencedirect.com/science/article/pii/S0925231207000355

[59] A. Joshi, F. Porikli, N. Papanikolopoulos, Breaking the interactive bottleneck in multi-class classification with active selection and binary feedback, in: Computer Vision and Pattern Recognition (CVPR), 2010 IEEE Conference on, 2010 , pp. $2995-3002$. doi:10.1109/CVPR.2010.5540047.

[60] H. Yu, C. Sun, W. Yang, X. Yang, X. Zuo, Al-elm: One uncertainty-based active learning algorithm using extreme learning machine, Neurocomputing 166 (2015) 140 - 150. doi:http://dx.doi.org/10.1016/j.neucom.2015.04.019. URL http://www.sciencedirect.com/science/article/pii/S0925231215004646

[61] S. Savarese, J. Winn, A. Criminisi, Discriminative object class models of appearance and shape by correlatons, in: Computer Vision and Pattern Recognition, 2006 IEEE Computer Society Conference on, Vol. 2, IEEE, 2006, pp. 2033-2040.

[62] M. Everingham, L. Van Gool, C. K. Williams, J. Winn, A. Zisserman, The pascal visual object classes (voc) challenge, International journal of computer vision 88 (2) (2010) 303-338.

[63] L. Fei-Fei, R. Fergus, P. Perona, Learning generative visual models from few training examples: An incremental bayesian approach tested on 101 object categories, Computer Vision and Image Understanding 106 (1) (2007) 59-70.

[64] M. Marszatek, C. Schmid, Accurate object localization with shape masks, in: Computer Vision and Pattern Recognition, 2007. CVPR '07. IEEE Conference on, 2007, pp. 1-8. doi:10.1109/CVPR.2007.383085.

[65] D. G. Lowe, Distinctive image features from scale-invariant keypoints, International journal of computer vision 60 (2) (2004) $91-110$.

[66] J. Tang, Z.-J. Zha, D. Tao, T.-S. Chua, Semantic-gap-oriented active learning for multilabel image annotation, Image Processing, IEEE Transactions on 21 (4) (2012) 2354-2360.

[67] O. A. Penatti, F. B. Silva, E. Valle, V. Gouet-Brunet, R. d. S. Torres, Visual word spatial arrangement for image retrieval and classification, Pattern Recognition 47 (2) (2014) 705-720. 
Zhipeng Ye is an Ph.D. candidate at the School of Computer Science and Technology, Harbin Institute of Technology. He receives Master degree of computer application technology of Harbin Institute of Technology in 2013. His research interest covers image processing and machine learning.

Peng Liu Associate professor at the School of Computer Science and Technology, HIT. He receives his Doctoral degree of microelectronics and solid state electronics of HIT in 2007. His research interest covers image processing, video processing, pattern recognition and design of VLSI circuit.

JiafengLiu Associate professorat the School of Computer Science andTechnology, Harbin Institute of Technology, and seniormember of China Computer Federation. He received hisPh. D. degree from Harbin Institute of Technology in 1996. His research interest covers pattern recognition, machinelearning, image processing, image understanding, and computer vision.

Wei Zhao Associate professor at the School of Computer Science and Technology. She receives her Doctoral degree of computer application technology of HIT in 2006. Her research interest covers pattern recognition, image processing, deep space target visual analysis.

Xianglong Tang Professor at the School of Computer Science and Technology, HIT. He receives Doctoral degree of computer application technology of HIT in 1995. His research interest covers pattern recognition, aerospace image processing, medical image processing and machine learning. 


\section{${ }^{*}$ Photo of the author(s)}

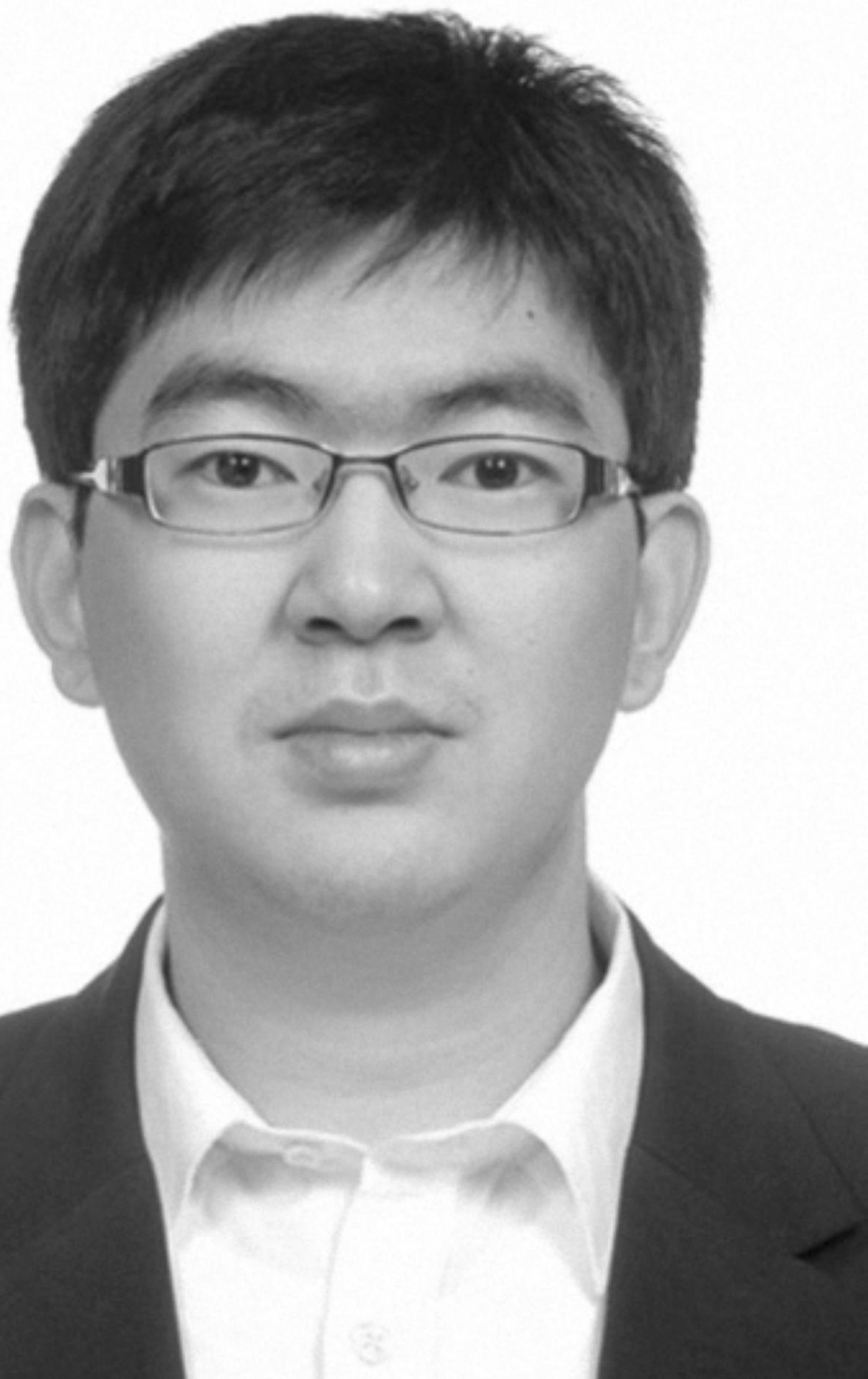


${ }^{*}$ Photo of the author(s)

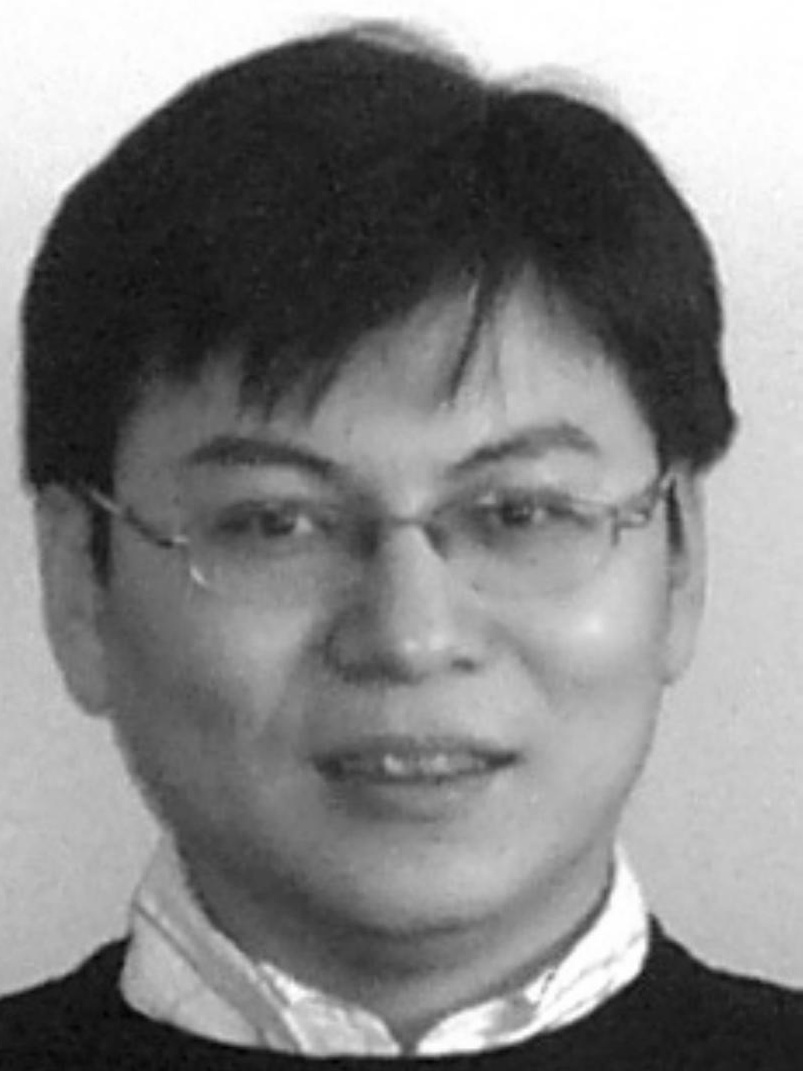




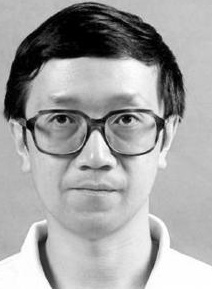




\section{${ }^{*}$ Photo of the author(s)}

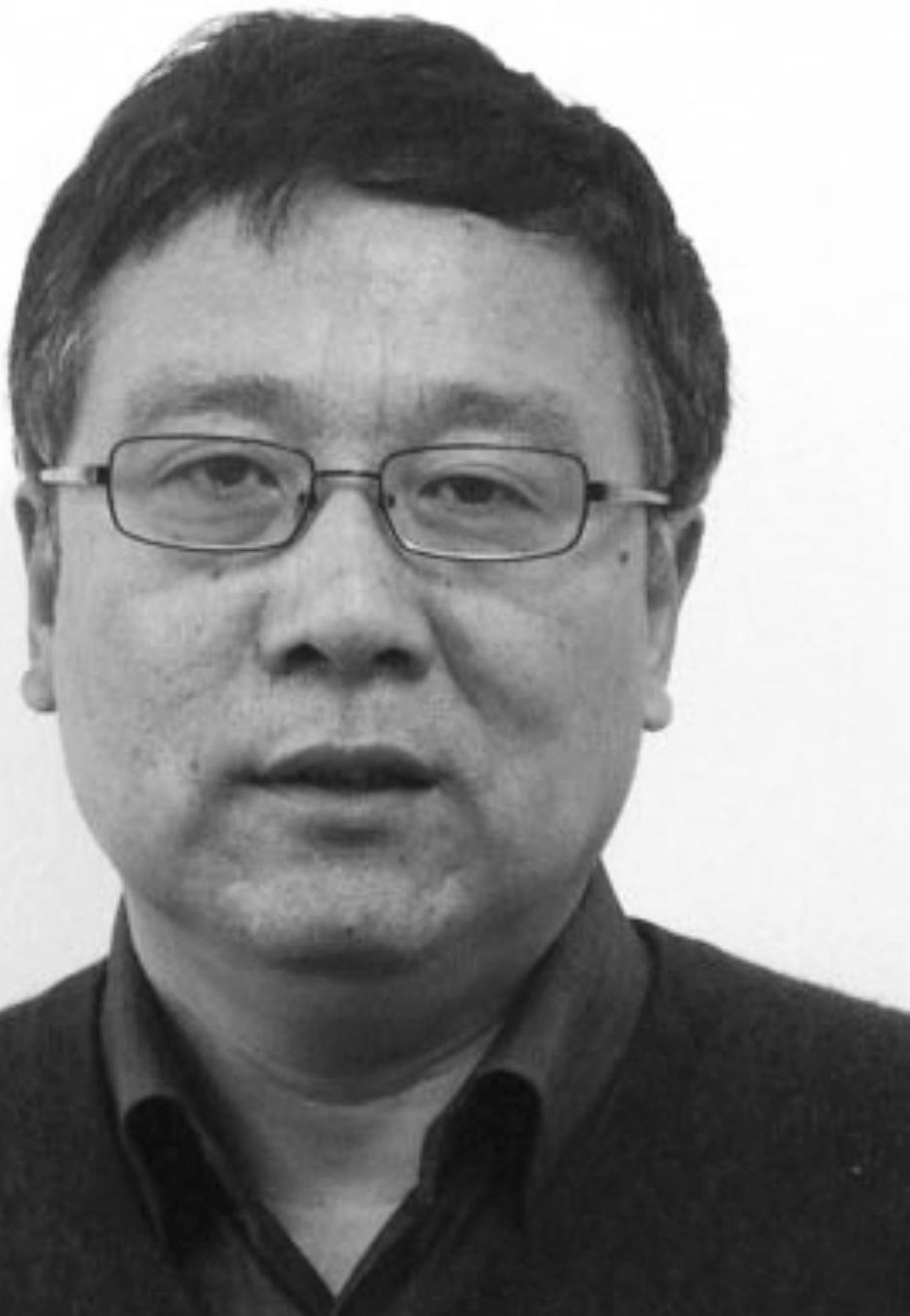


